\title{
Study on Pulse Monitor System of WBAN
}

\author{
Liu Hongyue ${ }^{1}$ \\ School of Information Engineering \\ Beijing Institute of Fashion Technology \\ Beijing, China \\ Liuhongyue_2013@163.com
}

\author{
Liu $\mathrm{Yi}^{3 *}$
}

Fashion Accessory Art and Engineering College

Beijing Institute of Fashion Technology

Beijing, China

gxyly@bift.edu.cn

\author{
Huo Tong ${ }^{2}$ \\ Fashion Accessory Art and Engineering College \\ Beijing Institute of Fashion Technology \\ Beijing, China \\ 1245322078@qq.com
}

\begin{abstract}
Wireless body area networks (WBANs) are small and short distance wireless sensor networks, widely used in the medical field. WBANs can realize whole-process tracking and intelligent services, which is of great significance for the dynamic monitoring of human information and disease prevention. The monitoring, acquisition and processing of human health information is the most direct method to measure individual health status and an important means to effectively prevent disease threats. Pulse is an important parameter of human body sign. In this paper, a pulse system for wireless body area network is designed. It is expected to realize daily monitoring of human pulse signals and provide remote medical services.
\end{abstract}

Keywords-WBAN; the pulse system; tele medicine

\section{THE RESEARCH BACKGROUND}

With the rapid development of material technology, sensor technology with computers and mobile phones as nodes has gradually turned to the Internet of things, which can be connected by wearable intelligent devices, household appliances and human bodies. WBAN is a typical representative of the Internet of things, which is composed of wireless biosensor nodes that logy and communication technology, the traditional Intern can be implanted in the human body or wearable [4]. WBAN can be applied in the fields of medical treatment, game entertainment, sports and other fields. It can realize short distance, low energy consumption and high reliability information transmission around the human body or in the human body. WBAN collects the required biological information by deploying the required biological sensor nodes on the patient and transmits it to the data platform through the coordinator [6]. Compared with the traditional medical system, this method on the one hand reduces the workload of medical staff to monitor patients and ensures the reliability and integrity of data records. On the other hand, it also avoids the restriction on patient activities of medical devices and their circuits.
In recent years, domestic and foreign experts are devoted to the development of non-invasive non-contact sensors [3]. An important feature of these sensors is that they do not cause injury to the body. And it can automatically eliminate the error of the instrument's own system. The sensor used in this paper is the photoelectric pulse sensor, which is a pulse sensor based on the photoelectric volume method[1]. The sensor works by monitoring the light transmittance at the tip of the finger to detect the pulse signal indirectly. The sensor has the advantages of simple structure, no damage, high precision and reusable [5].

\section{THE DESIGN THOUGHT}

In this paper constructs a wearable system to detect the pulse signal of human body. The pulse signal of human body is detected and the data collected by sensor is transmitted by wireless transmission technology. Through the communication network of the mobile phone, information interaction with the first aid center and the user's family is realized [4]. The overall design block diagram of WBAN is shown in Fig. 1. The system mainly collects the pulse information of the human body by placing relevant sensor equipment on the human body surface. When the sensor collects the human body sign parameters, it sends the data to the portable smartphone device through wireless transmission. Finally, the data is transmitted to the database transmitted to the remote medical service center through the wireless communication of smartphone devices. The medical staff sorted out and analyzed the data and gave feedback.

The node has the characteristics of miniaturization and low power consumption. It has signal acquisition and processing capability and wireless communication capability. The sensor module is responsible for collecting signals and processing and calculating accordingly [7]. The pulse of human body is in 
ceaseless beat pulse beat can cause the blood flow inside the body. This causes changes in light transmittance.

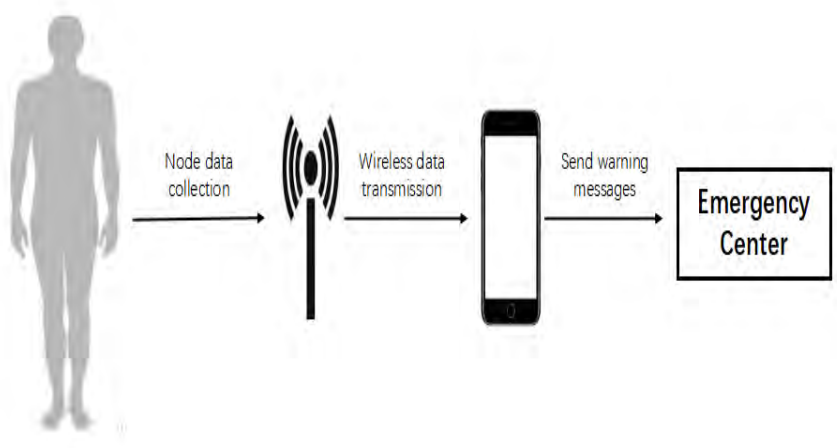

Fig. 1. Overall design block diagram of WBAN

The sensor used in this system is photoelectric sensor. Because light transmittance is constantly changing, when the fingertips are covered with the photoelectric sensor, the pulse signals it detects are constantly changing [1]. Since bluetooth technology is characterized by stable operation, low power, simple equipment and little harm to human body, it is selected as the communication method between node and terminal equipment.

\section{HARDWARE SYSTEM DESIGN}

In order to realize the whole function of wireless body area network, the key node of human body should be collected first. In this paper, infrared photoelectric sensor is used to generate pulse signal [2]. After amplification and shaping, the signal is input into the microcontroller for corresponding control, so as to conveniently and quickly measure the pulse rate within one minute. The overall design of the system is composed of STC89C52, key, digital tube, photoelectric sensor, clock module, operation and put. The system is equipped with four buttons to set the upper and lower pulse Numbers. When exceeding the range, the MCU will drive the buzzer to sound. The diagram of hardware circuit system is shown in Fig. 2.

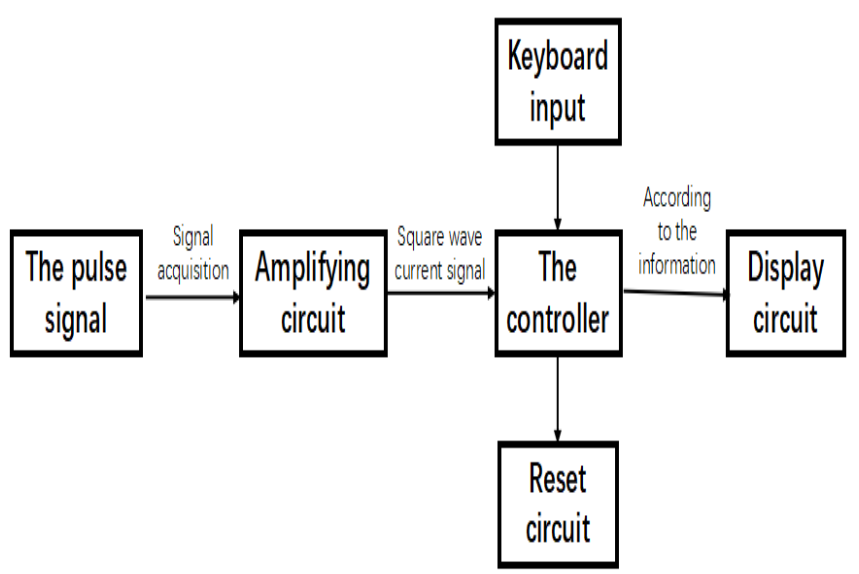

Fig. 2 Hardware system flow chart

Collection of pulse signals: this system collects the pulse signal using the photoelectric sensor. Its light source is usually a light-emitting diode with a selective wavelength of oxygen and hemoglobin in arterial blood [8]. Arterial pulsation causes changes in congestion volume and light transmittance when the beam passes through the body's peripheral blood vessels. At this point, the light reflected by human tissue is received by the photoelectric converter and transformed into an electrical signal, which is then amplified and output. Because the signal amplitude is small and easy to be interfered, the filter circuit and the amplifier circuit need to be added for back-end processing.

Adopting amplifying and filtering circuits: Pulse signal output is very weak, generally at the micron level. And the output signal is usually accompanied by very large noise interference. So we use LM358 to set up an amplification and filtering circuit. The adopting amplifying and filtering circuits is shown in Fig. 3.

Design of filter shaping circuit: The weak sinusoidal signal collected needs to be amplified and rectified and turned into square wave signal for processing in the circuit [9].

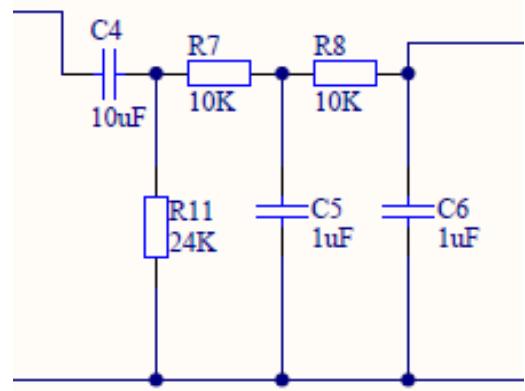

Fig. 3 Adopting amplifying and filtering circuits

The hysteresis voltage comparator is used to improve the anti-interference ability. Among them, LM358 is used for integrated operational amplifier. Circuit design is shown in Fig. 4.

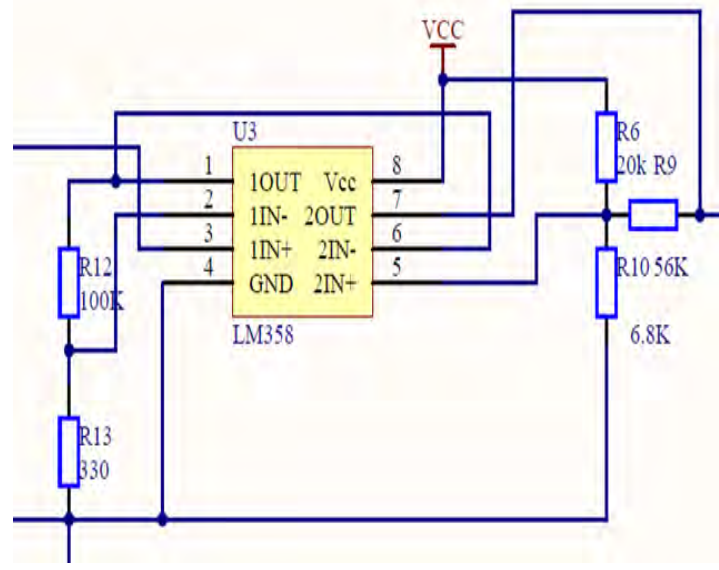

Fig. 4 Circuit design 


\section{SOFTWARE SYSTEM DESIGN}

The system has designed four key keys: measure key, set key, add key and subtract key. The upper and lower limits of the pulse measurement range can be set by setting the key in the system[6]. Increase or decrease alarm values by adding and subtracting keys. When the measurement key is pressed it triggers an interruption and begins the process of measuring the pulse. Fig. 5. is the main program flow chart [9].

Use a counter and add one to each timer interrupt trigger counter. The judgment is added to the external interrupt and is calculated only if the counter is greater than 5 , and the interrupt is ignored if it is less than 5. Five pulse intervals were continuously measured, and the expected pulse, converted to one minute, was displayed.

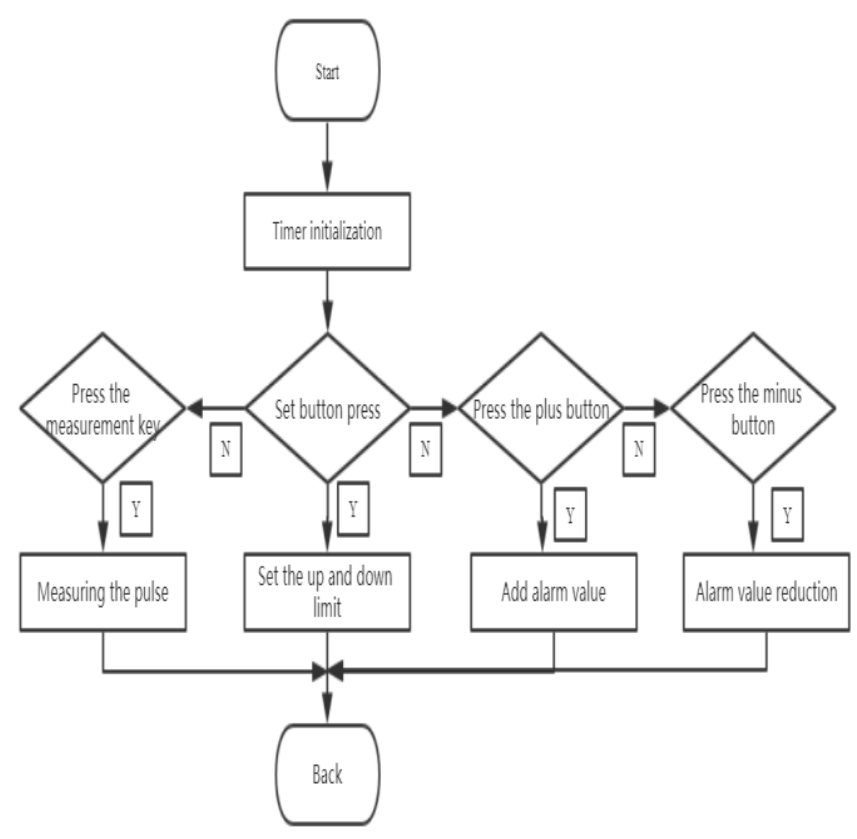

Fig. 5 Main program flow chart

The core part of the design of pulse system software is the process of pulse detection and calculation. Fig. 6 is the flow chart of pulse calculation. In the main function, the timer interrupt triggering time is set to $50 \mathrm{~ms}$ to start external interrupt, but the timer interrupt is not started at this time. When the signal of level hopping is received, the external interrupt is triggered. Turn on timer interrupt.

Pulse monitor system is a multi-functional digital design, so its program is more complex, so there are relatively many problems in the programming and debugging. difficulty of this design lies in the part of software coding and debugging. This part of the software is written in $\mathrm{C}$ language, which is relatively simple and seems to have a clear, clear and understandable context. And debugging, modification is more convenient. When writing, each module can be written independently. Each module is represented by a function and only needs to be called when necessary. Finally, each module is connected.

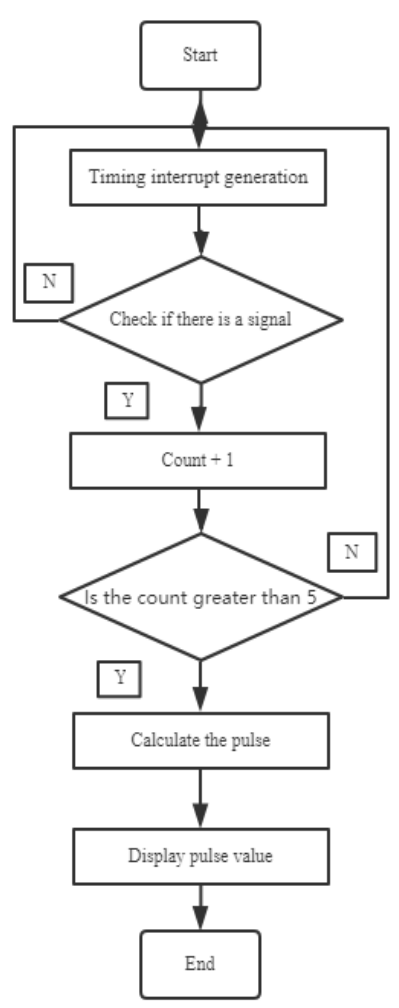

Fig. 6. Flow chart of pulse calculation

\section{SySTEM DEBUGGING}

This pulse meter system is a multifunctional digital design. In order to make the system run and work stably, hardware debugging and software debugging are carried out respectively according to the system design scheme. The debugging of the system hardware circuit is mainly to detect whether the circuit has leakage welding, circuit breaking, virtual welding, short circuit and whether it can work normally after starting up [7]. The main problems encountered during software debugging are as follows:

\section{A. After loading the program, the digital tube shows flashing and uneven brightness.}

First, the delay of the call is gradually modified to solve the display flash problem. Secondly, because this work makes the number displayed in the dynamic scanning mode and the dynamic scanning is very fast, so the human naked eye cannot see it. However, if the last value is not blocked on return, it will appear very bright. So the mask is added after the display and finally the problem is solved. 
B. when the user presses the button, the value read by the single-chip computer is wrong with the value set.

Re-check the connection of the matrix keyboard circuit and re-establish a new correspondence.

\section{RESULT ANALYSIS}

Four people were randomly selected to use the pulse system and the stethoscope to measure and record human pulse in the morning, at noon, at night and after exercise. The results are shown in Table 1.

TABLE I. MEASURED DATA AND ACTUAL DATA

\begin{tabular}{|c|c|c|c|c|c|}
\hline \multicolumn{2}{|c|}{} & Morning & Noon & Night & $\begin{array}{c}\text { After } \\
\text { exercise }\end{array}$ \\
\hline \multirow{2}{*}{1} & Measured & 87 & 79 & 74 & 96 \\
\cline { 2 - 6 } & Aactual & 90 & 76 & 73 & 92 \\
\hline \multirow{2}{*}{2} & Measured & 79 & 81 & 74 & 89 \\
\cline { 2 - 6 } & Aactual & 76 & 84 & 76 & 91 \\
\hline \multirow{3}{*}{3} & Measured & 76 & 72 & 75 & 88 \\
\cline { 2 - 6 } & Aactual & 74 & 76 & 80 & 92 \\
\hline \multirow{2}{*}{4} & Measured & 89 & 85 & 74 & 97 \\
\cline { 2 - 6 } & Aactual & 85 & 84 & 70 & 95 \\
\hline
\end{tabular}

The recorded results were compared using the mapping software, and the pulse number measured by the pulse system was compared with that measured by the stethoscope. There was a certain error between the two, but the curve trend was basically consistent. Fig. 7 shows partial measurement results
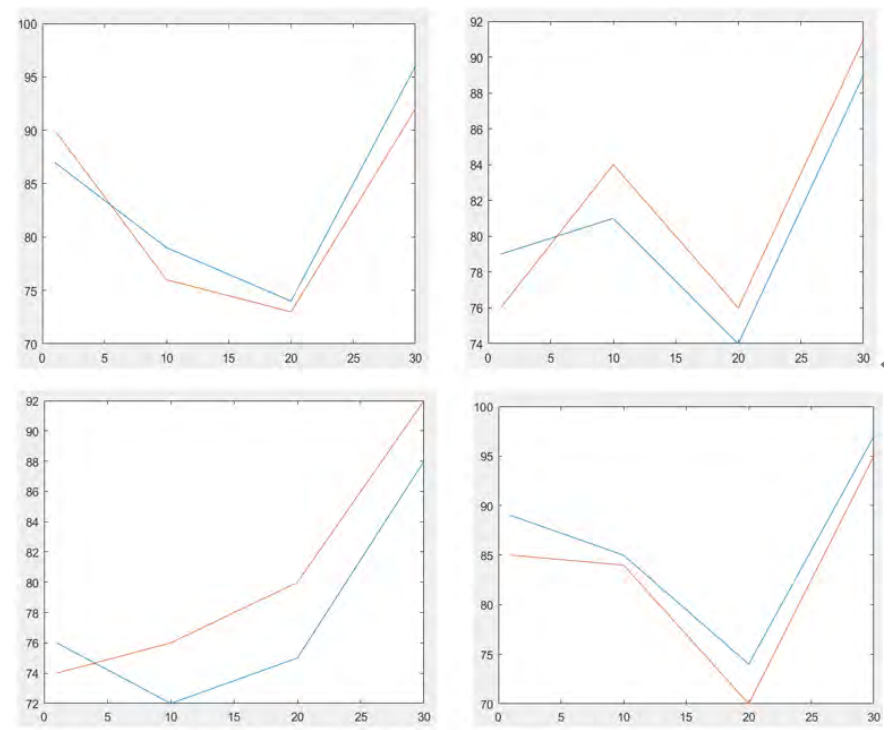

Fig. 7. Partial results

The designed pulse meter can realize the function of measuring pulse and is small and easy to carry. In the experiment, put the finger belly gently on the photoelectric sensor and wait patiently. When the indicator lights flicker uniformly, the value of the pulse is displayed on the screen. If the finger is pressed too tightly or not pressed properly, the measurement will be affected. Because the sensor is more sensitive the value will float up and down [3]. The upper and lower bounds of the pulse value are set by pressing the button. The buzzer driver module will give an alarm when the setting is out of range. Finally, the measurement results are displayed on the liquid crystal.

\section{ACKNOWLEDGMENT}

This research was financially supported by Beijing Institute of Fashion Technology College of Special Plan Young Topnotch Talent Project(BIFTBJ201803), Beijing Institute of Fashion Technology College Student Research Training Program (2018), Beijing Institute of Fashion Technology Teaching Reform and Innovation Key Program (ZDJG1709), Fang Zhi Zhi Guang China National Textile And Apparel Council Higher Education Teaching Reform and Innovation Program (2017-08), Beijing Municipal Education Commission Science and Technology Program on the surface of the project (SQKM201710012006), Innovative design of garment ergonomics and apparel functions Beijing key Laboratory (KYTG02170202), and Open Project of Digital and Interactive Media Key Laboratory (KF2017-04).

\section{REFERENCES}

[1] Liu Y, Liu D, Yue G. BGMM: A Body Gauss-Markov Based Mobility Model for Body Area Networks[J]. Tsinghua Science and Technology, 2018, 23(3):277-287.

[2] Otto, C. A., Jovanov, E., \& Milenkovic, A. (2006). A WBAN-based System for Health Monitoring at Home. Ieee/embs International Summer School on Medical Devices and BIOSENSORS (pp.20-23). IEEE. Zhao Yingjie. Perfect illustration. Introduction to Arduino interactive design. Beijing: Science Press, 2014.7.

[3] Cheng, S. H., \& Huang, C. Y. (2013). Coloring-based inter-wban scheduling for mobile wireless body area networks. IEEE Transactions on Parallel \& Distributed Systems, 24(2), 250-259. Fei Si technology research and development center, MATLAB6.5 aided image processing, electronic industry press, 2003.1

[4] Verbiest, J. R., \& Vandenbosch, G. A. E. (2006). A novel small-size printed tapered monopole antenna for uwb wban. IEEE Antennas Wireless Propag Lett, 5(1), 377-379. Sun Dejun editor in chief. Easy to learn and play Arduino. Arduino.: Chemical Industry Press, 2016.10

[5] Liao, P. (2004). Reform of the experimental teaching of single chip microcomputer. Research \& Exploration in Laboratory. Chen Ming, principles and examples of neural network, Tsinghua University press, 2013.3.

[6] Ma, J. (2004). Design of using single-chip microcomputer to control the ttemperature. Computer Automated Measurement \& Control, 12(12), 1219-1216.

[7] Zhu, Y. X., \& You, J. (2002). Exploration on reform of single-chip microcomputer experiment teaching. Laboratory Research \& Exploration.

[8] Cragon, H. G. (1980). The Elements of Single-Chip Microcomputer Architecture. IEEE Computer Society Press.

[9] Ren, X. R. (2003). Reliability design of single chip microcomputer system. Computer Automated Measurement \& Control. 